\title{
GEOMETRIC DIMENSION OF VECTOR BUNDLES OVER LENS SPACES
}

BY

\section{DENIS SJERVE}

1. Introduction. Throughout $p$ will denote an odd prime. Let $q_{1}, \ldots, q_{n+1}$ be relatively prime to $p$ and let $Z_{p}$ denote the cyclic group with generator $t$ and relation $t^{p}=1$. If $\theta=\exp (2 \pi \sqrt{ }(-1) / p)$, then we can define a free smooth action of $Z_{p}$ on the sphere $S^{2 n+1}$ by $t^{k}\left(z_{1}, \ldots, z_{n+1}\right)=\left(\theta^{k q_{1}} z_{1}, \ldots, \theta^{k q_{n+1}} z_{n+1}\right)$, where $\left(z_{1}, \ldots, z_{n+1}\right)$ is a complex $n+1$ tuple representing a point of $S^{2 n+1}$. The orbit space of this action is called a (generalized) lens space and is denoted by $L^{2 n+1}$. It is a compact, connected, orientable manifold of dimension $2 n+1$ and the quotient map $\pi: S^{2 n+1} \rightarrow L^{2 n+1}$ is a $p$-fold covering. If all the $q_{k}$ are equal then $L^{2 n+1}$ is known as a standard lens space.

Suppose $\xi$ is a stable vector bundle over a finite $C W$ complex $X$. Then $\xi$ may be interpreted as the homotopy class of a map $X \rightarrow B O$, where $B O$ is the classifying space for the infinite orthogonal group, and the geometric dimension of $\xi$ is the least nonnegative integer $k$ such that the following lifting problem may be solved:

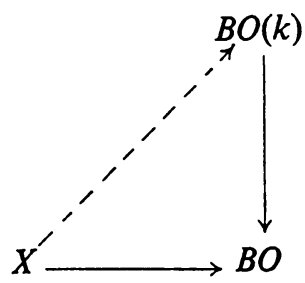

We write g. $\operatorname{dim} \xi$ for the geometric dimension of $\xi$.

If we let $P_{i}$ denote either the $i$ th Pontrjagin class or its $\bmod p$ reduction and if $c$ is any integer satisfying $4 c+1 \equiv 0(\bmod p)$, then our main theorems are:

TheOREM A. If $\xi \in(K O) \sim\left(L^{2 n+1}\right) \cap \operatorname{ker} \pi^{*}$, then $\operatorname{g.dim} \xi \leqq 2[n / 2]+1$.

TheOREM B. Suppose $n \geqq 2, p \geqq[n / 2]+3$, and $\xi \in(K O) \sim\left(L^{2 n+1}\right) \cap \operatorname{ker} \pi^{*}$. Then g. $\operatorname{dim} \xi \leqq 2[n / 2]$ if, and only if, there exists a cohomology class $\bar{u} \in H^{2[n / 2]}\left(L^{2 n+1} ; Z_{p}\right)$ such that $c P_{[n / 2]}(\xi)+\bar{u}^{2}=0$.

THEOREM C. (i) $L^{2 n+1}$ immerses in $R^{2 n+2[n / 2]+2}$ for all $n$ and all odd primes $p$;

(ii) if all the $q_{k}$ are equal, $n \geqq 2$, and $p \geqq[n / 2]+3$, then $L^{2 n+1}$ immerses in $R^{2 n+2[n / 2]+1}$ if, and only if,

is a quadratic residue $\bmod p$;

$$
(-1)^{[n / 2]}\left(\begin{array}{c}
n+[n / 2] \\
n
\end{array}\right)
$$

Received by the editors July $31,1967$. 
(iii) if all the $q_{k}$ are equal and

$$
\left(\begin{array}{c}
n+[n / 2] \\
n
\end{array}\right) \not \equiv 0(\bmod p)
$$

then $L^{2 n+1}$ does not immerse in $R^{2 n+2[n / 2]}$.

Therefore Theorem $\mathbf{C}$ solves the immersion problem for lens spaces with no twisting (i.e., all the $q_{k}$ equal) if the prime $p$ is sufficiently large.

Recently there have been published papers on the immersion problem for standard lens spaces. In particular see [2], [3], [4], and [7]. In [4] the case $p=3$ is treated and in [3] the following is proved: if $p \geqq 5$ and $n=\alpha p^{k}+\beta p^{1}$, where $\alpha, \beta, k, 1$ must satisfy certain restrictions, then $L^{2 n+1}$ does not immerse in $R^{2 n+2[n / 2]+1}$. This result does not overlap with Theorem $\mathrm{C}$.

Uchida's result [7] also applies to the standard lens spaces and says that $L^{2 n+1}$ immerses in $R^{2 n+2[n / 2]+4}$. The technique used in proving Theorems $\mathrm{A}$ and $\mathrm{B}$ is similar to that used by Uchida. The difference lies in the treatment of the top dimensional obstruction in the Postnikov resolution of the lifting problem:

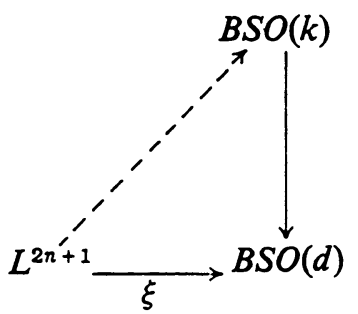

where $k=2[n / 2]$ or $2[n / 2]+1$ and $d$ is a sufficiently large integer. The fiber is the Stiefel manifold $V_{d, d-k}$ and therefore we must know something about their homotopy groups. What we need is stated in $\$ 2$. To the best of my knowledge this result does not appear in the literature, even though I am sure it is well known. Therefore, the proof is omitted.

Finally I would like to express my thanks to Professors E. Spanier and P. E. Thomas of Berkeley for their help.

2. Preliminary lemmas. Let $J$ denote any finite abelian group whose order is relatively prime to $p$ and let $\rho: Z$ or $Z \oplus J \rightarrow Z_{p}$ denote $\bmod p$ reduction. The following lemma is well known:

LEMMA (2.1). (i) $H^{*}\left(L^{2 n+1} ; Z_{p}\right)$ is a truncated polynomial algebra in the even dimensions on a two dimensional generator;

(ii) $H^{i}\left(L^{2 n+1} ; Z\right) \cong Z$ if $i=0,2 n+1$,

$$
\cong Z_{p} \text { if } i \text { even and } 2 \leqq i \leqq 2 n \text {, }
$$

$\cong 0$ otherwise.

Moreover there is a two dimensional generator $x$ such that in the even dimensions $H^{*}\left(L^{2 n+1} ; Z\right)$ is a polynomial algebra truncated by $x^{n+1}=0$; 
(iii) $H^{i}\left(L^{2 n+1} ; J\right)=0$ for $0<i<2 n+1$;

(iv) $\pi^{*}: H^{2 n+1}\left(L^{2 n+1} ; J\right) \rightarrow H^{2 n+1}\left(S^{2 n+1} ; J\right)$ is an isomorphism;

(v) $\rho_{*}: H^{2 i}\left(L^{2 n+1} ; Z\right)$ or $H^{2 i}\left(L^{2 n+1} ; Z \oplus J\right) \rightarrow H^{2 i}\left(L^{2 n+1} ; Z_{p}\right)$ is an isomorphism if $0<2 i<2 n+1$.

This lemma could be proved by using the cell decomposition given in [5]. For the next lemma let $V_{n, n-q}$ be the Stiefel manifold of $n-q$ frames in $n$-space. Denote by $\bar{n}$ (resp. $\bar{q}$ ) the greatest (resp. least) odd integer $\leqq n$ (resp. $\geqq q$ ) and denote by $S$ the product $S^{2 \ell+1} \times S^{2 \ell+5} \times \cdots \times S^{2 \pi-3}$, where we must include the factor $S^{n-1}$ if $n$ is even and the factor $S^{q}$ if $q$ is even. If $\bar{n}=\bar{q}$ replace $S^{2 \ell+1} \times \cdots \times S^{2 \pi-3}$ by a point. Putting $n_{1}$ equal to the least dimension of all the spheres in $S$ we have:

LEMMA (2.2). (i) For all $i, \pi_{i}\left(V_{n, n-q}\right)$ and $\pi_{i}(S)$ are isomorphic modulo finite groups;

(ii) if $n_{1}>2$, then the p-primary part of $\pi_{i}\left(V_{n, n-q}\right)$ is zero for $i<n_{1}+2 p-3$.

3. Proof of Theorem C. Let $\nu: L^{2 n+1} \rightarrow B O$ be the stable normal bundle of $L^{2 n+1}$. Because $\pi: S^{2 n+1} \rightarrow L^{2 n+1}$ is a $p$-fold covering we see that $\pi^{*}(\nu)=0$. Therefore Theorem A applies and g. $\operatorname{dim} \nu \leqq 2[n / 2]+1$. By a well-known theorem of Hirsch (see [1]) this proves (i).

According to [5] the total Pontrjagin class of the tangent bundle $\tau$ of $L^{2 n+1}$ is given by

$$
P(\tau)=\left(1+q_{1}^{2} x^{2}\right) \cdots\left(1+q_{n+1}^{2} x^{2}\right)
$$

where $x$ is as in (2.1). If all the $q_{k}=q$ we get $P(\tau)=\left(1+q^{2} x^{2}\right)^{n+1}$. Since there is no 2-torsion in $H^{*}\left(L^{2 n+1} ; Z\right)$ this implies that

$$
P(\nu)=\sum_{i \geqq 0}(-1)^{i}\left(\begin{array}{c}
n+i \\
i
\end{array}\right) q^{2 i} x^{2 i} .
$$

In particular we have

$$
P_{[n / 2]}(\nu)=(-1)^{[n / 2]}\left(\begin{array}{c}
n+[n / 2] \\
{[n / 2]}
\end{array}\right) q^{2[n / 2]} x^{2[n / 2]} .
$$

If $L^{2 n+1}$ immerses in $R^{2 n+2[n / 2]}$ we must have $P_{i}(v)=0$ for $i \geqq[n / 2]$. This proves (iii).

Now assume all the $q_{k}=q, n \geqq 2$, and $p \geqq[n / 2]+3$. Theorem B applies so that g. $\operatorname{dim} \nu \leqq 2[n / 2]$ if, and only if, there exists $\bar{u} \in H^{2[n / 2]}\left(L^{2 n+1} ; Z_{p}\right)$ so that

$$
c(-1)^{[n / 2]}\left(\begin{array}{c}
n+[n / 2] \\
{[n / 2]}
\end{array}\right) q^{2[n / 2]} \bar{x}^{2[n / 2]}+\bar{u}^{2}=0,
$$

where $\bar{x}$ is the mod $p$ reduction of $x$. But $\bar{x}$ generates the even dimensions of the truncated polynomial algebra $H^{*}\left(L^{2 n+1} ; Z_{p}\right)$ and therefore we can write $\bar{u}=a \bar{x}^{[n / 2]}$. Thus g. $\operatorname{dim} \nu \leqq 2[n / 2]$ if, and only if, there exists an integer $a$ satisfying

$$
c(-1)^{[n / 2]}\left(\begin{array}{c}
n+[n / 2] \\
n
\end{array}\right) q^{2[n / 2]}+a^{2} \equiv 0(\bmod p) .
$$


By simple number theory this is equivalent to

$$
(-1)^{[n / 2]}\left(\begin{array}{c}
n+[n / 2] \\
n
\end{array}\right)
$$

being a quadratic residue mod $p$. This proves (ii).

4. Proof of Theorem A. Consider the lifting problem in (1.1) for $k=2[n / 2]+1$. If $V$ denotes the fiber then (2.2) implies that $\pi_{i}(V)$ is finite with no " $p$-part" for $i \leqq 2 n$. Therefore, according to (2.1), in the Postnikov resolution we encounter only one possible nonzero obstruction:

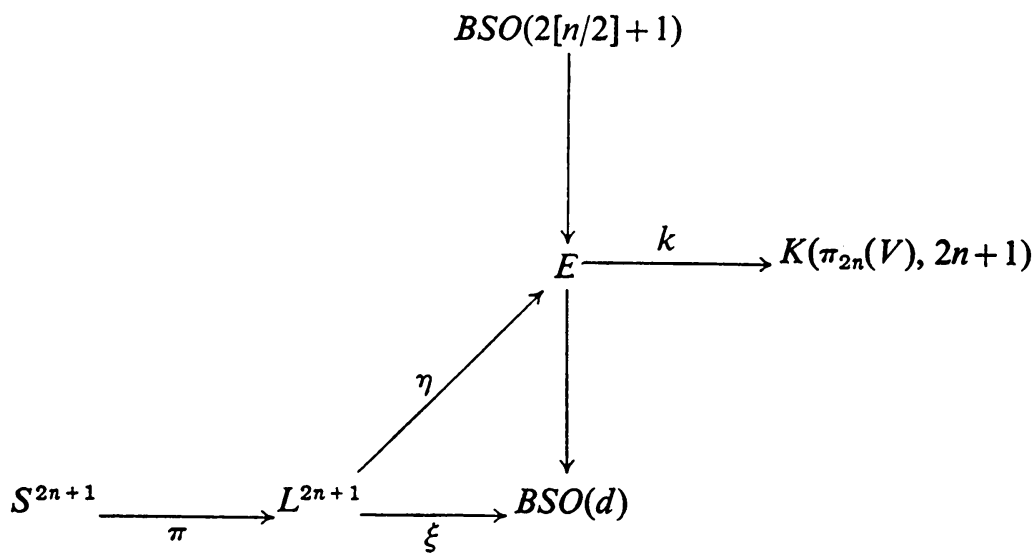

$\eta$ lifts $\xi$ up to $E$ and the last obstruction to lifting $\xi$ is $\eta^{*}(k) \in H^{2 n+1}\left(L^{2 n+1} ; \pi_{2 n}(V)\right)$. Since $\xi \circ \pi$ is trivial it must lift all the way to $B S O(2[n / 2]+1)$, and in particular, up to $E$. Moreover, its lifting up to $E$ must be unique and is therefore $\eta \circ \pi$. Since $\xi \circ \pi$ lifts past $E$ so does $\eta \circ \pi$ and this implies $\pi^{*} \eta^{*}(k)=0$. But (2.1) now gives $\eta^{*}(k)=0$. This proves Theorem A.

5. Proof of Theorem B. For convenience put $m=[n / 2]$. Then we are assuming $\xi$ is a real stable vector bundle over $L^{2 n+1}$ and that $\pi^{*}(\xi)$ is trivial and

$$
p \geqq m+3, \quad n \geqq 2 .
$$

Put $B=B S O(2 n+3)$ and $B^{\prime}=B S O(2 m)$ and let $p: B \rightarrow B^{\prime}$ be the standard fibration with fiber the Stiefel manifold $V=V_{2 n+3,2 n+3-2 m}$. As mentioned in the introduction to prove Theorem B we study the Postnikov resolution of the lifting problem:

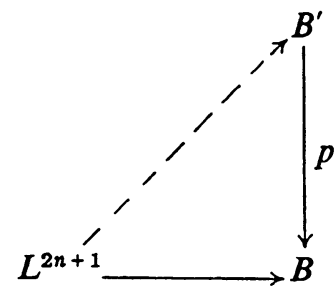


The following facts are well known and will be used implicitiy in what follows:

(1) $H^{*}(B S O(2 k+1) ; Z) \cong Z\left[P_{1}, \ldots, P_{k}\right] \oplus T$;

(2) $H^{*}(B S O(2 k) ; Z) \cong Z\left[P_{1}, \ldots, P_{k}, \chi_{2 k}\right] \oplus T$ with the relation $P_{k}=\chi_{2 k}^{2}$;

(3) $H^{*}\left(B S O(2 k+1) ; Z_{p}\right) \cong Z_{p}\left[P_{1}, \ldots, P_{k}\right]$;

(4) $H^{*}\left(B S O(2 k) ; Z_{p}\right) \cong Z_{p}\left[P_{1}, \ldots, P_{k}, \chi_{2 k}\right]$ with the relation $P_{k}=\chi_{2 k}^{2}$; where $\chi_{2 k}$ is the Euler class and $T$ is a subring consisting of elements of order 2.

The fiber $V$ of $p: B \rightarrow B^{\prime}$ is $2 m-1$ connected and $\pi_{2 m}(V) \cong Z$. Thus there is a fundamental class $v \in H^{2 m}(V ; Z)$ and the first Postnikov invariant is $k_{0}=\tau(v)$ $\in H^{2 m+1}(B ; Z)$, where $\tau$ is the transgression in the fibration $V \rightarrow B^{\prime} \stackrel{p}{\rightarrow} B$. Inducing a principal fibration over $B$ with $k_{0}$ as classifying map we get the commutative diagram

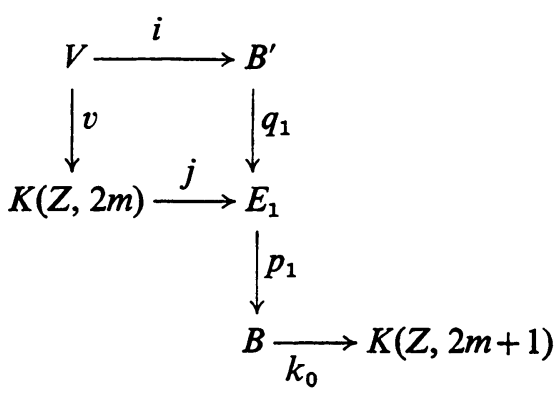

LEMMA (5.2). $k_{0}=\delta w_{2 m}$, where $w_{2 m} \in H^{2 m}\left(B ; Z_{2}\right)$ is a Stiefel-Whitney class and $\delta$ is the Bockstein coboundary associated to the coefficient sequence $0 \rightarrow Z \rightarrow Z$ $\rightarrow Z_{2} \rightarrow 0$.

Proof. Let $B^{\prime \prime}=B S O(2 m+1)$. Then we have a commutative diagram of fibrations

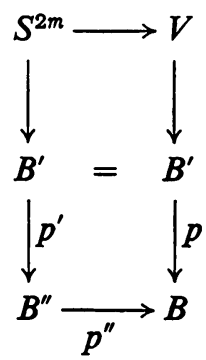

where $p^{\prime}, p^{\prime \prime}$ are the natural fibrations. If $s^{*} \in H^{2 m}\left(S^{2 m} ; Z\right)$ is the fundamental class and $\tau^{\prime}$ the transgression for $p^{\prime}$, then

$$
p^{\prime \prime *}\left(k_{0}\right)=p^{\prime *} \tau(v)=\tau^{\prime}\left(s^{*}\right)=\chi_{2 m+1} \in H^{2 m+1}\left(B^{\prime \prime} ; Z\right),
$$

the Euler class (see [6]). The fiber of $p^{\prime \prime}$ is $2 m$ connected and therefore

$$
p^{\prime \prime *}: H^{2 m+1}(B ; Z) \rightarrow H^{2 m+1}\left(B^{\prime \prime} ; Z\right)
$$

is a monomorphism. Now $\delta w_{2 m}=\chi_{2 m+1}$ is a relation in $H^{*}\left(B^{\prime \prime} ; Z\right)$ and thus $p^{\prime \prime *}\left(\delta w_{2 m}\right)=p^{\prime \prime *}\left(k_{0}\right)$, i.e., $k_{0}=\delta w_{2 m}$. Q.E.D. 
Now consider the Serre exact sequence of $p: B^{\prime} \rightarrow B$ :

$\cdots \longrightarrow H^{2 m}(B ; Z) \longrightarrow H^{2 m}\left(B^{\prime} ; Z\right) \longrightarrow H^{2 m}(V ; Z) \stackrel{\tau}{\longrightarrow} H^{2 m+1}(B ; Z) \longrightarrow \cdots$.

This reduces to

$$
\cdots \longrightarrow H^{2 m}(B ; Z) \stackrel{p^{*}}{\longrightarrow} H^{2 m}\left(B^{\prime} ; Z\right) \stackrel{i^{*}}{\longrightarrow} Z \longrightarrow Z_{2} \longrightarrow 0
$$

because $H^{2 m}(V ; Z) \cong Z$ is generated by $v, \tau(v)=k_{0} \neq 0$, and $2 k_{0}=0$. Since $\chi_{2 m}$ $\in H^{2 m}\left(B^{\prime} ; Z\right)$ generates coker $p^{*}$ in dimension $2 m$ we see that $i^{*}\left(\chi_{2 m}\right)= \pm 2 v$. If need be we change the sign of $v$ so that

$$
i^{*}\left(\chi_{2 m}\right)=2 v \text {. }
$$

This will not change the Postnikov resolution since $k_{0}=-k_{0}$.

Now consider the higher Postnikov invariants:

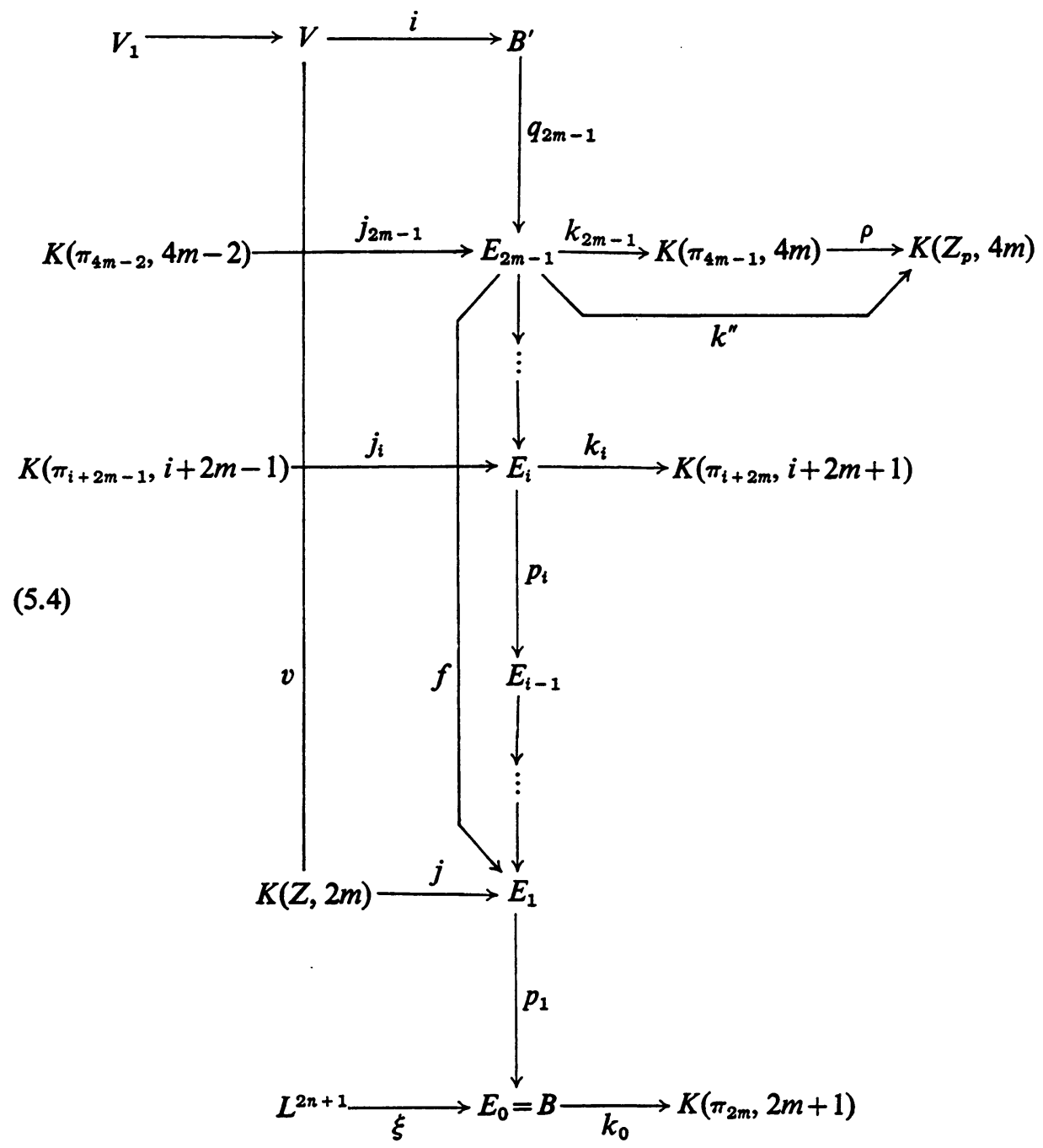


where $\pi_{i}=\pi_{i}(V)$. Let $f$ be the composite $E_{2 m-1} \rightarrow \cdots \rightarrow E_{1}$. Now from (2.2) it follows that

(5.5) (i) $\pi_{i}$ has no " $p$-part" for $i<4 m+3$;

(ii) $\pi_{i}$ is finite for $2 m<i<4 m-1$ and $4 m-1<i<4 m+3$;

(iii) $\pi_{4 m-1}$ and $\pi_{4 m+3}$ are isomorphic to $Z$ modulo finite groups.

Recall that $\rho: \pi_{4 m-1} \rightarrow Z_{p}$ is $\bmod p$ reduction. Then define $k^{\prime \prime}$ to be $\rho_{*}\left(k_{2 m-1}\right)$.

In the remainder of this section, unless stated otherwise, all cohomology groups will have $Z_{p}$ coefficients.

LEMMA (5.6). There is a unique class $k \in H^{4 m}\left(E_{1}\right)$ such that $f^{*}(k)=k^{\prime \prime}$. Moreover $q_{1}^{*}(k)=0$.

Proof. From (5.4) we see that $f$ is a composition of fibrations whose fibers are Eilenberg-MacLane spaces $K\left(\pi_{r}, r\right), 2 m+1 \leqq r \leqq 4 m-2$. By (5.5) it follows that the $\bmod p$ cohomology of these fibers is trivial and therefore $f^{*}: H^{*}\left(E_{1}\right) \cong H^{*}\left(E_{2 m-1}\right)$ by some Serre exact sequences. Finally $q_{1}^{*}(k)=q_{2 m-1}^{*}\left(k^{\prime \prime}\right)=\rho_{*} q_{2 m-1}^{*}\left(k_{2 m-1}\right)=0$. Q.E.D.

The reason for introducing $k$ is given by:

Lemma (5.7). $\xi$ lifts past $E_{2 m-1}$ in the Postnikov resolution (5.4) if, and only if, there exists a lifting $\eta: L^{2 n+1} \rightarrow E_{1}$ so that $\eta^{*}(k)=0$.

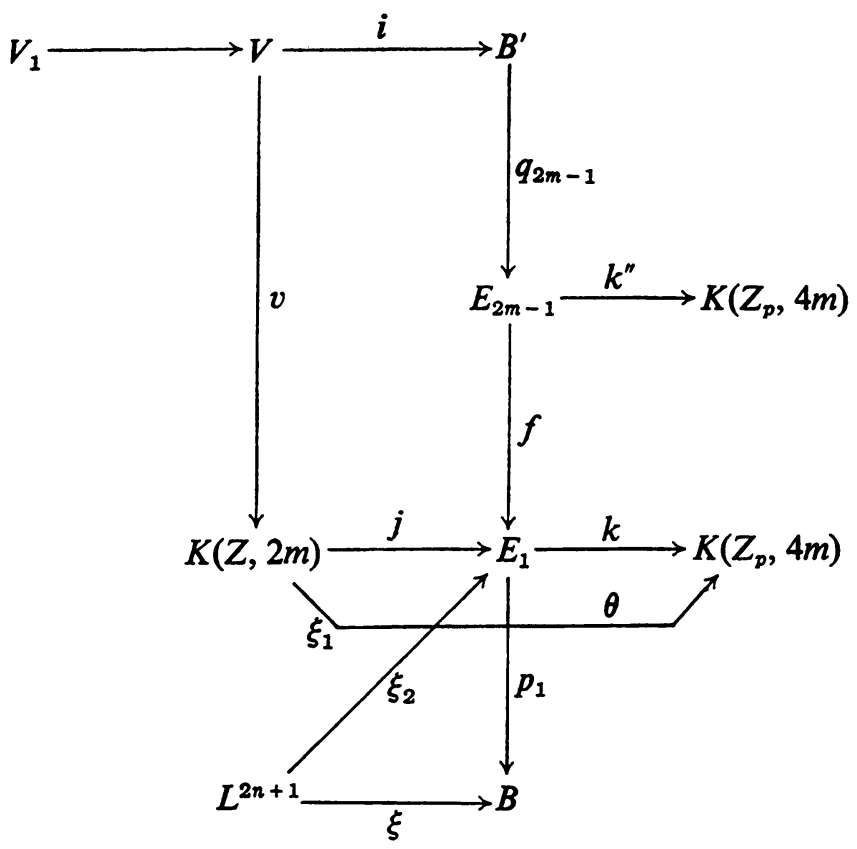

Proof. First note that $\xi$ lifts to $E_{1}$ since $\xi^{*}\left(k_{0}\right)=0$ by (2.1). The obstructions to lifting $\xi$ up to $E_{2 m-1}$ lie in the groups $H^{i}\left(L^{2 n+1} ; \pi_{i-1}\right)$ for $2 m+2 \leqq i \leqq 4 m-1$. But all these groups are zero according to (2.1) and (5.5) so that $\xi$ lifts to $\bar{\eta}: L^{2 n+1}$ $\rightarrow E_{2 m-1}$. Let $\eta=f \circ \bar{\eta}: L^{2 n+1} \rightarrow E_{1}$. Then $\xi$ lifts past $E_{2 m-1}$ if, and only if, we can 
choose $\bar{\eta}$ so that $\bar{\eta}^{*}\left(k_{2 m-1}\right)=0$. But $\rho_{*}: H^{4 m}\left(L^{2 n+1} ; \pi_{4 m-1}\right) \cong H^{4 m}\left(L^{2 n+1} ; Z_{p}\right)$ and therefore $\bar{\eta}^{*}\left(k_{2 m-1}\right)=0$ if, and only if, $\bar{\eta}^{*}\left(k^{\prime \prime}\right)=0$. Now $f^{*}$ is an isomorphism for $Z_{p}$ coefficients and so $\bar{\eta}^{*}\left(k^{\prime \prime}\right)=0$ if, and only if, $\eta^{*}(k)=0$. This shows that if $\xi$ lifts past $E_{2 m-1}$ then such a map $\eta$ exists. Conversely, given $\eta$ we can lift it to $E_{2 m-1}$ and proceed as above. Q.E.D.

The situation now is shown in the preceding equation (5.8).

Define a cohomology operation $\theta$ by $\theta=j^{*}(k) \in H^{4 m}\left(Z, 2 m ; Z_{p}\right) \cdot p_{1}: E_{1} \rightarrow B$ is a principal fibration and therefore there is an action of the fiber $K(Z, 2 m)$ on the total space $E_{1}, \mu_{1}: K(Z, 2 m) \times E_{1} \rightarrow E_{1}$ (see [6]). This induces an action on the homotopy level $\mu_{1}:[\circ, K(Z, 2 m)] \times\left[\circ, E_{1}\right] \rightarrow\left[\circ, E_{1}\right]$. If $\xi_{1}, \xi_{2}$ are liftings of $\xi$ to $E_{1}$ then they differ by this action, i.e., there exists a class $u \in H^{2 m}\left(L^{2 n+1} ; Z\right)$ such that $\xi_{2}$ is the composite

$$
L^{2 n+1} \stackrel{d}{\longrightarrow} L^{2 n+1} \times L^{2 n+1} \stackrel{u \times \xi_{1}}{\longrightarrow} K(Z, 2 m) \times E_{1} \stackrel{\mu_{1}}{\longrightarrow} E_{1}
$$

where $d$ is diagonal. Let $\iota \in H^{2 m}\left(Z, 2 m ; Z_{p}\right)$ be the fundamental class. In $\S 6$ it is proved that $\theta=\iota^{2}$. If we use the notation $\bar{u}$ for $u$ reduced $\bmod p$ we have:

LEMMA (5.9). (i) $\mu_{1}^{*}(k)=1 \otimes k+\iota^{2} \otimes 1+\imath \otimes x$ for some $x \in H^{2 m}\left(E_{1}\right)$;

(ii) $\xi_{2}^{*}(k)-\xi_{1}^{*}(k)=\bar{u}^{2}+u \cup \xi_{1}^{*}(x)$.

Proof. Let $s_{1}: K(Z, 2 m) \rightarrow K(Z, 2 m) \times E_{1}$ and $s_{2}: E_{1} \rightarrow K(Z, 2 m) \times E_{1}$ be the canonical inclusions with respect to chosen base points. Then $\mu_{1} \circ s_{1} \simeq j$ and $\mu_{1} \circ S_{2} \simeq 1_{E_{1}}$ (see [6]). By the Künneth formula $\mu_{1}^{*}(k)$ must contain $1 \otimes k+\iota^{2} \otimes 1$. Because of (5.1) it follows that $H^{i}\left(Z, 2 m ; Z_{p}\right)=0$ for $2 \dot{m}<i<4 m$ and $H^{4 m}\left(Z, 2 m ; Z_{p}\right)$ $\cong Z_{p}$ generated by $\imath^{2}$. Thus. there is at most one more term in $\mu_{1}^{*}(k)$ and that is a cross term $\iota \otimes x$. Finally

$$
\begin{aligned}
\xi_{2}^{*}(k) & =\left(u \cdot \xi_{1}\right)^{*}(k)=d^{*}\left(u \times \xi_{1}\right)^{*} \mu_{1}^{*}(k) \\
& =d^{*}\left(u \times \xi_{1}\right)^{*}\left(1 \otimes k+\iota^{2} \otimes 1+\imath \otimes x\right)=\xi_{1}^{*}(k)+\bar{u}^{2}+u \cup \xi_{1}^{*}(x) . \quad \text { Q.E.D. }
\end{aligned}
$$

Defining a cohomology operation $\varphi$ by $\varphi=j^{*}(x) \in H^{2 m}\left(Z, 2 m ; Z_{p}\right)$ we get by a similar proof:

LEMMA (5.10). (i) $\mu_{1}^{*}(x)=1 \otimes x+\varphi \otimes 1$;

(ii) $\xi_{2}^{*}(x)-\xi_{1}^{*}(x)=\varphi(u)$.

If $\xi_{1}, \xi_{2}$ are liftings of $\xi$ to $E_{1}$ then $\xi_{2}=u \cdot \xi_{1}$ and $\xi_{1}=-u \cdot \xi_{2}$. Thus (5.9) gives

$$
\bar{u}^{2}-u \cup \xi_{2}^{*}(x)=\xi_{1}^{*}(k)-\xi_{2}^{*}(k)=-\left(\xi_{2}^{*}(k)-\xi_{1}^{*}(k)\right)=-\bar{u}^{2}-u \cup \xi_{1}^{*}(x),
$$

i.e., $2 \bar{u}^{2}=u \cup\left(\xi_{2}^{*}(x)-\xi_{1}^{*}(x)\right)=u \cup \varphi(u)$. Since this is true for all $u \in H^{2 m}\left(L^{2 n+1} ; Z\right)$ we get

$$
\varphi=2 \iota \text {. }
$$

(5.10) now becomes 
LEMMA (5.12). (i) $\mu_{1}^{*}(x)=1 \otimes x+2 \imath \otimes 1$;

(ii) $\xi_{2}^{*}(x)-\xi_{1}^{*}(x)=2 \bar{u}$.

The importance of $x$ is now clear since two liftings are homotopic if, and only if, $\xi_{1}^{*}(x)=\xi_{2}^{*}(x)$. Notice also that there is a unique $\xi_{1}$ satisfying $\xi_{1}^{*}(x)=0$. In all that follows $\xi_{1}$ will denote this particular lifting. Summarizing we have proved:

LemMA (5.13). (i) $\mu_{1}^{*}(k)=1 \otimes k+\iota^{2} \otimes 1+\iota \otimes x$ for some $x \in H^{2 m}\left(E_{1}\right)$;

(ii) $\varphi=j^{*}(x)=2 \iota$;

(iii) $\xi_{2}^{*}(k)-\xi_{1}^{*}(k)=\bar{u}^{2}$;

(iv) $\xi_{2}^{*}(x)=2 \bar{u}$.

In $\S 7$ it is proved that $\xi_{1}^{*}(k)=c P_{m}(\xi)$, where $c$ is as in $\S 1$. From (5.13) and (5.7) we immediately get

THEOREM (5.14). $\xi$ lifts past $E_{2 m-1}$ in the Postnikov resolution (5.4) if, and only if, there exists $u \in H^{2 m}\left(L^{2 n+1} ; Z\right)$ so that $c P_{m}(\xi)+\bar{u}^{2}=0$. If such a $u$ exists then $\xi_{2}$ $= \pm u \cdot \xi_{1}$ are the liftings of $\xi$ that lift past $E_{2 m-1}$.

Now it is simple to finish the proof of Theorem B. Assume there exists a cohomology class $u$ satisfying (5.14). Then $\xi$ at least lifts past $E_{2 m-1}$. The proof is completed exactly as Theorem $\mathrm{A}$ in $\$ 4$.

6. Proof of $\theta=\iota^{2}$. Define $v$ to be the composite

$$
K(Z, 2 m) \times B^{\prime} \stackrel{1 \times q_{1}}{\longrightarrow} K(Z, 2 m) \times E_{1} \stackrel{\mu_{1}}{\longrightarrow} E_{1} .
$$

According to [6] there is a homotopy commutative diagram of fibrations

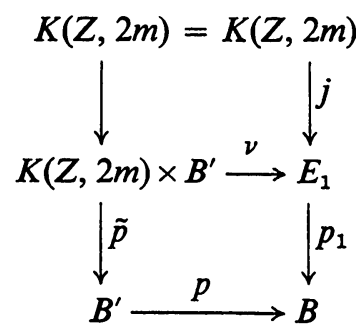

where $\tilde{p}$ is the product fibration. Finally there is the relative Serre exact sequence

$$
\begin{aligned}
\cdots \longrightarrow H^{r}\left(E_{1}\right) \stackrel{\nu^{*}}{\longrightarrow} H^{r}\left(K(Z, 2 m) \times B^{\prime}\right) \stackrel{\tau_{0}}{\longrightarrow} H^{r+1}\left(B, B^{\prime}\right) \\
\stackrel{1^{*}}{\longrightarrow} H^{r+1}\left(E_{1}\right) \longrightarrow \cdots \stackrel{\nu^{*}}{\longrightarrow} H^{4 m}\left(K(Z, 2 m) \times B^{\prime}\right),
\end{aligned}
$$

where $\tau_{0}$ is the relative transgression and 1 is the composite $E_{1} \stackrel{p_{1}}{\longrightarrow} B \subset\left(B, B^{\prime}\right)$. The next lemma is technical and will be needed later.

LEMMA (6.1). In dimensions $\leqq 4 m+3, H^{*}\left(B, B^{\prime}\right)$ is a free $H^{*}(B)$ module on one generator $\delta\left(\chi_{2 m}\right) \in H^{2 m+1}\left(B, B^{\prime}\right)$, where $\delta$ is the coboundary for the pair $\left(B, B^{\prime}\right)$. 
Proof. Note that $p^{*}: H^{*}(B) \rightarrow H^{*}\left(B^{\prime}\right)$ is $1-1$ in these dimensions and therefore the following sequence is exact for $q \leqq 4 m+2$ :

$$
0 \longrightarrow H^{q}(B) \stackrel{p^{*}}{\longrightarrow} H^{q}\left(B^{\prime}\right) \stackrel{\delta}{\longrightarrow} H^{q+1}\left(B, B^{\prime}\right) \longrightarrow 0 .
$$

Now coker $p^{*}$ is a free $H^{*}(B)$ module on one generator $\chi_{2 m} \in H^{2 m}\left(B^{\prime}\right)$ in dimensions $\leqq 4 m+3$. Since $\delta$ is an $H^{*}(B)$ morphism the result follows. Q.E.D.

CoRollaRY (6.2). $\nu^{*}: H^{i}\left(E_{1}\right) \rightarrow H^{i}\left(K(Z, 2 m) \times B^{\prime}\right)$ is $1-1$ for $i=2 m, 4 m$.

Since $H^{4 m}\left(Z, 2 m ; Z_{p}\right) \cong Z_{p}$ is generated by $\iota^{2}$ we certainly have $\theta=a \iota^{2}$ for some integer $a$. Just as we proved (5.11) we can show that $\varphi=2 a$.

THEOREM (6.3). $\theta \neq 0$, i.e., $a \neq 0(\bmod p)$.

Proof. Assume the contrary, i.e., $\theta=0$ and $\varphi=0$. By the Serre exact sequence of the fibration $p_{1}: E_{1} \rightarrow B$ and the definition of $\varphi$ there is $y \in H^{2 m}(B)$ such that $x=p_{1}^{*}(y)$. Now the transgression $\tau_{0}: H^{q}\left(K(Z, 2 m) \times B^{\prime}\right) \rightarrow H^{q+1}\left(B, B^{\prime}\right)$ is an $H^{*}(B)$ morphism where $H^{*}\left(K(Z, 2 m) \times B^{\prime}\right)$ is made into an $H^{*}(B)$ module by the map

$$
K(Z, 2 m) \times B^{\prime} \stackrel{\tilde{p}}{\longrightarrow} B^{\prime} \stackrel{p}{\longrightarrow} B
$$

(see [6]). Also $\tau_{0}$ can be defined on a subset of $H^{4 m}\left(K(Z, 2 m) \times B^{\prime}\right)$ containing image $\nu^{*}$. Moreover $\tau_{0}$ is zero on image $\nu^{*}$. Thus

$$
\nu^{*}(k)=\left(1 \times q_{1}\right)^{*} \mu_{1}^{*}(k)=\left(1 \times q_{1}\right)^{*}(1 \otimes k+\iota \otimes x)=\iota \otimes p^{*}(y)
$$

since $\mu_{1}^{*}(k)=1 \otimes k+\theta \otimes 1+\iota \otimes x$ (see the proof of (5.9)) and $q_{1}^{*}(k)=0$. This means that $\tau_{0}\left(\iota \otimes p^{*}(y)\right)=0$. But $\tau_{0}\left(\iota \otimes p^{*}(y)\right)=\tau_{0}(y \cdot \iota \otimes 1)$ (by the definition of the $H^{*}(B)$ module structure $)=y \cdot \tau_{0}(\iota \otimes 1)$. Therefore it follows that either $y=0$ or $\tau_{0}(\iota \otimes 1)=0$.

Assume $y=0$. Then $x=p_{1}^{*}(\dot{y})=0$ and $\nu^{*}(k)=\iota \otimes p^{*}(y)=0$, i.e., $k=0$ by (6.2). This implies that the $\bmod p$ reduction of the Postnikov invariant $k_{2 m-1}$ is zero. Let $V_{2 m-1}$ be the fiber of $q_{2 m-1}$ in (5.4). Then $V_{2 m-1}$ is $4 m-2$ connected and $\pi_{4 m-1}\left(V_{4 m-1}\right) \cong \pi_{4 m-1}$. If $v \in H^{4 m-1}\left(V_{2 m-1} ; \pi_{4 m-1}\right)$ is the fundamental class, then $k_{2 m-1}$ is the transgression of $v$ in the fibration $q_{2 m-1}$. Since $\rho_{*}\left(k_{2 m-1}\right)=0$ we see that $\rho_{*}(v)$ transgresses to zero, which implies by the Serre exact sequence of $q_{2 m-1}$, that $\rho_{*}(v)$ is the image of a class in $H^{4 m-1}\left(B^{\prime}\right)$. But $H^{4 m-1}\left(B^{\prime}\right)=0$ and thus $\rho_{*}(v)=0$. This is a contradiction since $\rho_{*}(v)$ can be identified with $\bmod p$ reduction in $\operatorname{Hom}\left(\pi_{4 m-1} ; Z_{p}\right)$.

Therefore $y \neq 0$ and $\tau_{0}(\iota \otimes 1)=0$. Then there exists $z \in H^{2 m}\left(E_{1}\right)$ such that $\nu^{*}(z)=\iota \otimes 1$. But

$$
\nu^{*}(z)=\left(1 \times q_{1}\right)^{*} \mu_{1}^{*}(z)=\left(1 \otimes q_{1}\right)^{*}\left(1 \otimes z+j^{*}(z) \otimes 1\right)=1 \otimes q_{1}^{*}(z)+j^{*}(z) \otimes 1 .
$$

Thus $q_{1}^{*}(z)=0$ and $j^{*}(z)=\iota$. But $q_{1}^{*}$ is an isomorphism in dimension $2 m$ and again we have a contradiction. Q.E.D. 
We have proved that $\theta=a c^{2}$, where $a \neq 0(\bmod p)$. Let $a^{\prime}$ be such that $a a^{\prime} \equiv 1(\bmod p)$. Then multiplication by $a^{\prime}$ in $Z_{p}$ induces a homotopy equivalence of $K\left(Z_{p}, 4 m\right)$ with itself. It is clear that as far as the obstructions to lifting $\xi$ are concerned we may replace $k$ by $a^{*}(k)$ as the Postnikov invariant, i.e., without loss of generality we may assume $\theta=\imath^{2}$.

7. Proof of $\xi_{1}^{*}(k)=c P_{m}(\xi)$. $\bmod p$ reduction of the first Postnikov invariant $k_{0}$ is zero since $H^{2 m+1}\left(B ; Z_{p}\right)=0$. Thus there is a commutative diagram of fibrations

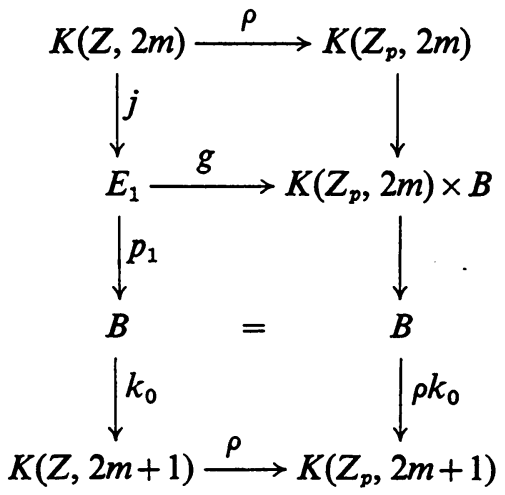

(7.2) Let $h: B^{\prime} \rightarrow K\left(Z_{p}, 2 m\right) \times B$ be the composite $g \circ q_{1}$ and let $F$ denote the fiber of $h$.

Then we have the commutative diagram

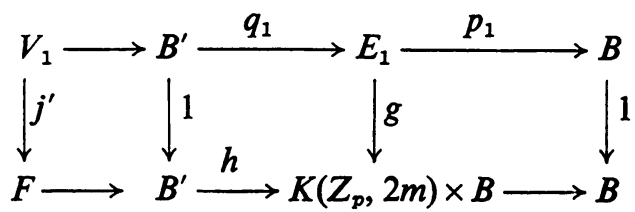

where $V_{1}=$ fiber $q_{1} . K\left(Z_{p}, 2 m\right)$ is an $H$-space via loop multiplication $\mu_{2}: K\left(Z_{p}, 2 m\right)$ $\times K\left(Z_{p}, 2 m\right) \rightarrow K\left(Z_{p}, 2 m\right)$. The action of the fiber on the total space of the trivial fibration induced by $\rho k_{0}$ is simply $\mu_{2} \times 1: K\left(Z_{p}, 2 m\right) \times K\left(Z_{p}, 2 m\right) \times B \rightarrow K\left(Z_{p}, 2 m\right)$ $\times B$ and we have the commutative diagram

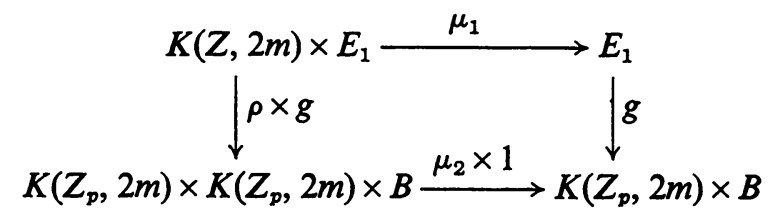

Let $\iota_{1} \in H^{2 m}\left(Z_{p}, 2 m ; Z_{p}\right)$ be the fundamental class.

LEMMA (7.5). (i) $h^{*}\left(\iota_{1} \otimes 1\right)=(1 / 2) \chi_{2 m}+p^{*}(y)$ for some $y \in H^{2 m}(B)$;

(ii) $h^{*}: H^{r}\left(K\left(Z_{p}, 2 m\right) \times B\right) \rightarrow H^{r}\left(B^{\prime}\right)$ is an isomorphism if $r<4 m$; 
(iii) $1 \otimes b+\iota_{1} \otimes b^{\prime}+\lambda \iota_{1}^{2} \otimes 1 \in H^{4 m}\left(K\left(Z_{p}, 2 m\right) \times B\right) \cap \operatorname{ker} h^{*}$, where $b \in H^{4 m}(B)$ and $b^{\prime} \in H^{2 m}(B)$, if and only if, $b^{\prime}+2 \lambda y=0$ and $2 y b^{\prime}+4 b+\lambda P_{m}=0$.

Proof. $h^{*}\left(\iota_{1} \otimes 1\right)=q_{1}^{*} g^{*}\left(\iota_{1} \otimes 1\right)$ and so $i^{*} h^{*}\left(\iota_{1} \otimes 1\right)=v^{*} j^{*} g^{*}\left(\iota_{1} \otimes 1\right)=v^{*} \rho^{*}\left(\iota_{1}\right)$ $=v^{*}(\iota)=\bar{v}=\rho_{*}(v)$. But we also have $i^{*}\left(\frac{1}{2} \chi_{2 m}\right)=\bar{v}$ according to (5.3). From the Serre exact sequence of $p: B^{\prime} \rightarrow B$ we get $h^{*}\left(\iota_{1} \otimes 1\right)=\frac{1}{2} \chi_{2 m}+p^{*}(y)$ for some $y \in H^{2 m}(B)$. This proves (i). (ii) and (iii) are short calculations. Q.E.D.

From the Serre exact sequence of $h$ we easily get:

Corollary (7.6).

$$
\begin{aligned}
& H^{r}(F) \cong Z_{p} \text { if } r=0,4 m-1 \text {, } \\
& \cong 0 \quad \text { if } 0<r<4 m-1 \text {. }
\end{aligned}
$$

According to (7.1) and (7.5) there exists uniquely $k^{\prime} \in H^{4 m}\left(K\left(Z_{p}, 2 m\right) \times B\right)$ such that

$$
h^{*}\left(k^{\prime}\right)=0 \text { and } j^{*} g^{*}\left(k^{\prime}\right)=\iota^{2} \text {, }
$$

namely $k^{\prime}=1 \otimes b+\iota_{1} \otimes b^{\prime}+\iota_{1}^{2} \otimes 1$, where $b^{\prime}=-2 y$ and $b=-\left(\frac{1}{4}\right) P_{m}+y^{2}$. Thus $g^{*}\left(k^{\prime}\right)$ satisfies $q_{1}^{*}\left(g^{*}\left(k^{\prime}\right)\right)=0$ and $j^{*}\left(g^{*}\left(k^{\prime}\right)\right)=\iota^{2}$. Both of these conditions are satisfied by $k$ and we would like to show that $g^{*}\left(k^{\prime}\right)=k$.

Let $V_{i}$ be the fiber of $q_{i}: B^{\prime} \rightarrow E_{i}$. Then we have the fibrations $V_{i+1} \rightarrow V_{i} \stackrel{v_{i}}{\rightarrow}$ $K\left(\pi_{2 m+i}, 2 m+i\right), i \geqq 0$, where $V_{0}=V, v_{0}=v$, and $v_{i} \in H^{2 m+i}\left(V_{i}, \pi_{2 m+i}\right)$ is the fundamental class. The maps $V_{i+1} \rightarrow V_{i}$ compose to give a map $V_{2 m-1} \rightarrow V_{1}$. From (5.5) it follows that this composition induces an isomorphism between $H^{*}\left(V_{1}\right)$ and $H^{*}\left(V_{2 m-1}\right)$. Therefore

$$
\begin{aligned}
H^{r}\left(V_{1}\right) & \cong Z_{p} & \text { if } r=0 \text { or } 4 m-1, \\
& \cong 0 & \text { if } 0<r<4 m-1,
\end{aligned}
$$

since $V_{2 m-1}$ is $4 m-2$ connected and $H^{4 m-1}\left(V_{2 m-1}\right) \cong \operatorname{Hom}\left(\pi_{4 m-1}, Z_{p}\right) \cong Z_{p}$.

From (7.3) we get the commutative diagram

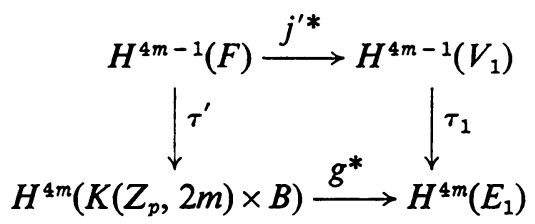

where $\tau_{1}, \tau^{\prime}$ are the respective transgressions. Since $h^{*}\left(k^{\prime}\right)=0$ the Serre exact sequence of $h$ says that there is $v^{\prime} \in H^{4 m-1}(F)$ such that $k^{\prime}=\tau^{\prime}\left(v^{\prime}\right)$. Thus $g^{*}\left(k^{\prime}\right)$ $=\tau_{1} j^{*}\left(v^{\prime}\right)$. Since $q_{1}^{*}(k)=0$ we see that $k$ is in the image of $\tau_{1}$ and therefore if we could show that $j^{\prime *}: H^{4 m-1}(F) \cong H^{4 m-1}\left(V_{1}\right)$ it would follow that $k$ is in the image of $g^{*}$. To do this we need only show that $g^{*}\left(k^{\prime}\right) \neq 0$. After a straightforward calculation based on (7.4) we get

$$
\nu^{*} g^{*}\left(k^{\prime}\right)=\imath \otimes \chi_{2 m}+\iota^{2} \otimes 1
$$

Because $k^{\prime}$ is the only class satisfying (7.7) we conclude $k=g^{*}\left(k^{\prime}\right)$. 
Now $\nu^{*}(k)=\left(1 \times q_{1}\right)^{*} \mu_{1}^{*}(k)=\left(1 \times q_{1}\right)^{*}\left(1 \otimes k+\iota^{2} \otimes 1+\iota \otimes x\right)=\iota^{2} \otimes 1+\iota \otimes q_{1}^{*}(x)$ since $q_{1}^{*}(k)=0$. Therefore we get

$$
q_{1}^{*}(x)=\chi_{2 m} .
$$

$h^{*}\left(\iota_{1} \otimes 1\right)=q_{1}^{*} g^{*}\left(\iota_{1} \otimes 1\right)=\frac{1}{2} \chi_{2 m}+p^{*}(y)$ by (7.5). Since $q_{1}^{*}$ is an isomorphism in dimension $2 m$ it follows that

$$
g^{*}\left(\iota_{1} \otimes 1\right)=\frac{1}{2} x+p_{1}^{*}(y) .
$$

Finally

$$
\begin{aligned}
\xi_{1}^{*}(k) & =\xi_{1}^{*} g^{*}\left(k^{\prime}\right)=\xi_{1}^{*} g^{*}\left(1 \otimes b+\iota_{1} \otimes b^{\prime}+\iota_{1}^{2} \otimes 1\right) \\
& =\xi^{*}(b)+\xi_{1}^{*} g^{*}\left(\iota_{1} \otimes 1\right) \xi^{*}\left(b^{\prime}\right)+\xi_{1}^{*} g^{*}\left(\iota_{1} \otimes 1\right)^{2} \\
& =\xi^{*}(b)+\xi^{*}(y) \xi^{*}\left(b^{\prime}\right)+\xi^{*}(y)^{2}=\xi^{*}\left(b+y b^{\prime}+y^{2}\right) \\
& =\xi^{*}\left(-\frac{1}{4} P_{m}\right)=c P_{m}(\xi) .
\end{aligned}
$$

A finAl REMARK. Since $(K O)^{\sim}\left(S^{2 n+1}\right)=0$ if $n \neq \equiv(\bmod 4)$ and $Z_{2}$ otherwise and since it can be shown that $(K O)^{\sim}\left(L^{2 n+1}\right)$ has order twice a power of $p$ if $n \equiv 0(\bmod 4)$ it follows that the restriction $\xi \in \operatorname{ker} \pi^{*}$ in Theorems $A$ and $B$ is not so restrictive.

\section{REFERENCES}

1. M. W. Hirsch, Immersions of manifolds, Trans. Amer. Math. Soc. 93 (1959), 242-267.

2. T. Kambe, The structure of $K_{\wedge}$-rings of the lens space and their applications, J. Math. Soc. Japan 18 (1966), 135-146.

3. T. Kobayashi, Non immersion theorems for lens spaces, J. Math. Kyoto Univ. 6 (1966), 91-108.

4. T. Kobayashi and R. Nakagawa, Non imbeddability of lens spaces mod 3, J. Math. Kyoto Univ. 5 (1966), 313-324.

5. J. Milnor, Whitehead torsion, Bull. Amer. Math. Soc. 72 (1966), 358-426.

6. E. Thomas, Seminar on fiber spaces, Lecture Notes in Math., No. 13, Springer-Verlag, Berlin, 1966.

7. F. Uchida, Immersions of lens spaces, Tôhoku Math. J. 18 (1966), 393-397.

Eidgenossische TeChNisChe Hochschule, ZURICH, SWITZERLAND 